\title{
DEGUM-Studie: Ultraschall mit Kontrastmittel erkennt Leberkrebs zuverlässig
}

Bösartige Tumoren in der Leber lassen sich nach der Injektion eines Kontrastmittels in einer Ultraschalluntersuchung frühzeitig diagnostizieren. In einer bundesweiten Studie der Deutschen Gesellschaft für Ultraschall in der Medizin e. V. (DEGUM) hat die Kontrastmittelsonografie bei Patienten mit Leberzirrhose in über $90 \%$ der Fälle Krebserkrankungen zuverlässig erkannt. Da Leberkrebs lange keine Symptome verursacht, raten Experten der DEGUM Risikopatienten zu regelmäßigen Ultraschalluntersuchungen.

Die Leberzirrhose ist die häufigste Ursache für Leberkrebs, an dem in Deutschland jährlich etwa 9000 Menschen erkranken. Zu den Risikopatienten zählen dabei Menschen mit einer chronischen Hepatitis-CVirusinfektion und diejenigen, die an einer nicht alkoholbedingten Fettlebererkrankung leiden. Alkoholkonsum ist laut RKI bei Frauen für $15 \%$ und bei Männern für $35 \%$ der Krebsneuerkrankungen der Leber verantwortlich. „Ultraschalluntersuchungen sind eine einfache und effektive Methode, um diese sogenannten hepatozellulären Karzinome frühzeitig zu erkennen“, sagt Professor Dr. med. Thomas Bernatik, Chefarzt der Medizinischen Klinik I der Kreisklinik Ebersberg bei München. Die Untersuchung belaste die Patienten anders als eine Computertomografie nicht durch ein Strahlenrisiko. Sie sei zudem wesentlich kostengünstiger als eine Kernspintomografie (MRT). Deshalb könne sie jederzeit wiederholt werden. „Dies kommt Patienten mit einer Leberzirrhose zugute, bei denen regelmäßige Kontrolluntersuchungen not- wendig sind, um einen Krebs frühzeitig zu erkennen“, so der DEGUM-Experte.

Mit einer einfachen Ultraschalluntersuchung kann der Arzt die Leberzirrhose beurteilen und verdächtige Knoten entdecken. Eine genaue Beurteilung ist jedoch erst mit einer Kontrastmittelsonografie (contrast enhanced ultrasound, CEUS) möglich. Das Kontrastmittel wird dabei über eine Armvene in den Körper geleitet. In die Leber gelangt es über die Leberarterien, die das Organ mit Sauerstoff und Nährstoffen versorgen. Das Kontrastmittel strömt auch in die Tumoren, die infolge ihrer guten Durchblutung im Ultraschall kontrastreicher erscheinen als die Umgebung. Der Kontrast entsteht, weil die Ultraschallwellen die gasgefüllten Mikrobläschen, aus denen das Kontrastmittel besteht, nicht durchdringen.

Ärzte können sich an 2 Algorithmen orientieren (CEUS-LI-RADS und ESCULAP), von denen einer - ESCULAP - in Deutschland entwickelt wurde. Die beiden Algorithmen geben dem Untersucher Hinweise, worauf er bei der Untersuchung achten muss. Die von der DEGUM unterstützte Studie hat den Nutzen der beiden Algorithmen in 43 Zentren an 321 Patienten untersucht. Nach der Gabe des Kontrastmittels erkannten die Ärzte 232 der 256 hepatozellulären Karzinome. Das Team um Professor Bernatik gibt die Sensitivität der Untersuchung mit 90,6\% an. Unter Verwendung des ESCULAP-Algorithmus konnte die Sensitivität sogar auf $94,2 \%$ gesteigert werden. Mit dem CEUSLI-RADS lag dieser Wert nur bei $64 \%$. Profes- sor Bernatik schließt aus den Ergebnissen, dass der ESCULAP-Algorithmus sich bewährt habe. Erfahrene Ärzte würden aber auch ohne ihn eine nahezu gleich hohe diagnostische Genauigkeit erzielen.

Auch für Dr. Thomas Müller vom St.-JosefsHospital Wiesbaden $\mathrm{GmbH}$ sind dies exzellente Ergebnisse. Die „CEUS HCC“-Studie habe erneut belegt, dass eine Ultraschalluntersuchung mit Kontrastmittel in der Lage sei, ein hepatozelluläres Karzinom zu erkennen. Auf Computer- und Kernspintomografie könne bei vielen Patienten verzichtet werden. Dass die meisten Ärzte auch ohne die Algorithmen die richtige Diagnose stellten, ist für Müller, der bei der DEGUM die Sektion Innere Medizin leitet, eine Bestätigung für die Fortbildungsinitiativen der DEGUM, die in Deutschland Ärzte in der Technik der Ultraschalluntersuchung fortbildet.

\section{Literatur:}

- Schellhaas B, Bernatik T, Bohle W et al. Contrast-Enhanced Ultrasound Algorithms (CEUS-LIRADS/ESCULAP) for the Noninvasive Diagnosis of Hepatocellular Carcinoma A Prospective Multicenter DEGUM Study. Ultraschall in Med 2020. doi:10.1055/a-1220-8561

- CEUS LI-RADS “Contrast-Enhanced UltraSound Liver Imaging Reporting and Data System"

- ESCULAP “Erlanger Synopsis for ContrastEnhanced Ultrasound for Liver Lesion Assessment in Patients at risk" 